\title{
Localization and Functional Characterization of Rat Kidney-specific Chloride Channel, CIC-K1
}

\author{
Shinichi Uchida, ${ }^{\star}$ Sei Sasaki, ${ }^{\star}$ Kosaku Nitta, ${ }^{\mathbf{5}}$ Keiko Uchida, ${ }^{\mathbf{5}}$ Shigeru Horita, $\mathbf{5}$ Hiroshi Nihei, ${ }^{\mathbf{5}}$ and Fumiaki Marumo* \\ *Second Department of Internal Medicine, Tokyo Medical and Dental University, School of Medicine. 1-5-45 Yushima, Bunkyo-ku, \\ Tokyo, 113, Japan; and ${ }^{\S}$ Department of Internal Medicine, Kidney Center, Tokyo Women's Medical College, 8-1 Kawada-cyo, \\ Shinjyuku, Tokyo, 162, Japan
}

\begin{abstract}
To investigate the physiological role of a kidney-specific chloride channel (ClC-K1), we sought to determine its exact localization by immunohistochemistry and its functional regulation using Xenopus oocyte expression system. The antiserum specifically recognized a $70-\mathrm{kD}$ protein in SDSPAGE of membrane protein from rat inner medulla and an in vitro translated ClC-K1 protein. Immunohistochemistry revealed that ClC-K1 was exclusively localized to the thin limb of Henle's loop in rat inner medulla. In comparison with the immunostaining with anti-aquaporin-CHIP antibody that only stains the descending thin limb of Henle's loop (tDL), ClC-K1 was found to be localized only in the ascending limb (tAL) which has the highest chloride permeability among nephron segments. Immunoelectron microscopy confirmed that the staining of ClC-K1 in tAL was observed in the region of both apical and basolateral plasma membranes. Expressed chloride current in Xenopus oocytes by ClC-K1 cRNA was regulated by extracellular pH and extracellular calcium. Furosemide inhibited the expressed current $\left(K_{i}=100 \mu \mathrm{M}\right)$, whereas $N$-ethyl-maleimide stimulated the current. These functional characteristics were consistent with the in vitro perfusion studies of chloride transport in tAL. The localization and the functional characteristics described here indicate that $\mathrm{ClC}-\mathrm{K} 1$ is responsible for the transepithelial chloride transport in tAL. (J. Clin. Invest. 1995. 95:104-113.) Key words: kidney epithelium • urinary concentration • immunohistochemistry • pH-regulated chloride channel $\cdot$ Xenopus oocytes
\end{abstract}

\section{Introduction}

Transepithelial chloride transport has been characterized in many tissues including kidney $(1,2)$. Two routes for transepithelial chloride transport have been postulated, namely transcellular and paracellular pathways. Transcellular chloride transport needs some specific transporters and/or channels for chloride ion in both apical and basolateral membranes. Numerous physi-

Address correspondence to Shinichi Uchida, Second Department of Internal Medicine, Tokyo Medical and Dental University, School of Medicine, 1-5-45 Yushima, Bunkyo-ku, Tokyo, 113, Japan. Phone: 3-38136111, x3658; FAX: 3-3818-7177.

Received for publication 12 July 1994 and in revised form 9 September 1994.

J. Clin. Invest.

(c) The American Society for Clinical Investigation, Inc.

0021-9738/95/01/0104/10 $\$ 2.00$

Volume 95, January 1995, 104-113 ological studies including patch clamp studies demonstrated the existence of chloride channels in kidney epithelia $(3,4)$, however the molecular basis of renal chloride transport remains poorly understood.

So far several putative chloride channels have been cloned and their functional characteristics have been examined (511). However no chloride channel except the cystic fibrosis transmembrane conductance regulator (CFTR) ${ }^{1}$ has been implicated in transcellular chloride transport. In kidney, the expression of CFTR was demonstrated but there is no symptom of kidney dysfunction in cystic fibrosis patients, suggesting that CFTR might not be involved in a major way in transepithelial chloride transport in kidney. There are, to our knowledge, four chloride channel clones at present whose expression in kidney have been confirmed $(6-8,10,11)$. The Madin-Darby canine kidney cells (MDCK) chloride channel cloned by expression cloning strategy have $\beta$ sheet structures which resemble the voltage dependent anion channel in mitochondria membrane (7). We showed its ubiquitous expression in non-epithelial tissues (12). Recently, this protein was found not to be a chloride channel itself but a regulator for a volume-regulated chloride channel (13). p64 was a chloride channel cloned from bovine kidney, but the injection of p64 cRNA into Xenopus oocytes did not elicit any chloride currents (6). It is likely that p64 represents the chloride channel in intracellular organelles (6). ClC-2 is a member of $\mathrm{ClC}$ chloride channel family originally isolated from heart and brain cDNA libraries and turned out to be expressed in various tissues including kidney (10). However, its functional characteristics did not fit for a chloride channel mediating transcellular chloride transport because it is not open at physiological membrane potential (10). It needs the stretch of the membrane to be open at the physiological membrane voltage, suggesting that it is involved in cell volume regulation (10).

We have recently isolated another member of $\mathrm{ClC}$ chloride channel family, ClC-K1 (11). ClC-K1 is exclusively expressed in kidney, especially in the inner medulla (11). Reverse transcription and polymerase chain reaction (RT-PCR) technique using isolated nephron segments revealed the main site of expression in kidney was the thin limb of Henle's loop (11). The thin ascending limb of Henle's loop was reported to have the highest chloride permeability among nephron segments (14) and there was indirect evidence that this high chloride permeability might be mediated by a chloride channel protein in the plasma membrane (15). Rapid $\mathrm{NaCl}$ exit from the thin ascending limb

1. Abbreviations used in this paper: $\mathrm{AQP}$, aquaporin; CFTR, cystic fibrosis transmembrane conductance regulator; MDCK, Madin-Darby canine kidney cells; NEM, $N$-ethyl-maleimide; RT, reverse transcription; tAL, ascending limb of Henle's loop; tDL, descending thin limb of Henle's loop. 
of Henle' loop is one of the major prerequisites for the models of urinary concentrating mechanisms in inner medulla (16-18). Based on these informations, $\mathrm{ClC}-\mathrm{K} 1$ could be involved in the transcellular chloride transport in the thin ascending limb of Henle's loop and the urinary concentrating mechanisms. RTPCR did not allow us to determine the cellular localization of $\mathrm{ClC}-\mathrm{K} 1$, even the exact localization of $\mathrm{ClC}-\mathrm{K} 1$ protein along nephron segments because the tiny segment like thin limb of Henle's loop can easily contaminate PCR samples of other nephron segments.

Here we demonstrate the cellular localization of ClC-K1 protein in kidney using polyclonal antiserum raised against the synthetic peptide as antigen. Furthermore, functional characterization of ClC-K1 in Xenopus oocytes and their comparison with the in vitro perfusion studies confirmed that ClC-K1 is a major chloride channel in tAL. Thus, we for the first time demonstrate the existence of transcellular chloride transport pathway via a chloride channel (ClC-K1) in kidney epithelia.

\section{Methods}

Preparation of antisera. Two oligopeptides corresponding to residues 1-15 and 604-618 were synthesized (peptide $N$ and peptide $C$, respectively). $5 \mathrm{mg}$ of each peptide was dissolved in water with $25 \mathrm{mg}$ of thyroglobulin and $50 \mathrm{mg}$ of carbodiimide was added to the mixture at $4^{\circ} \mathrm{C}$. After overnight conjugation at $4^{\circ} \mathrm{C}$, these conjugates were mixed with an equal volume of complete Freund's adjuvant, injected subcutaneously into rabbits ( $200 \mu \mathrm{g}$ peptide per rabbit). Booster injections were administered after 2.5-wk intervals with the same amount of protein, using incomplete Freund's adjuvant. The titers of sara were monitored by enzyme-linked immunosorbent assay (ELISA). Briefly, the synthetic peptides were fixed on 96 -well plates $(0.5 \mu \mathrm{g} /$ well $)$ and the serial dilutions of antisera were applied on the plates. An alkaliphophatase-conjugated anti-rabbit IgG antibody was used for the detection of bound antibodies to the antigen peptides. ELISA titer was expressed as a maximum dilution of antiserum where OD by the diluted serum was significantly higher than that by the negative control without serum.

In vitro translation of $\mathrm{ClC}-\mathrm{K} 1.2 \mu \mathrm{g}$ of ClC-K1 cRNA was synthesized using T7 RNA polymerase as previously described (11) and used for in vitro translation using rabbit reticulolysate with ${ }^{35} \mathrm{~S}$-methionine $(1,200 \mathrm{Ci} / \mathrm{mmol}$; Amersham International, Buckinghamshire, UK) in the presence or absence of canine pancreatic microsome membrane (Promega Corp., Madison, WI). The synthesized protein was denatured at $65^{\circ} \mathrm{C}$ for $1 \mathrm{~min}$, resolved in SDS-polyacrylamide gel (4-20\% gradient gel) electrophoresis, and visualized by autoradiography.

Immunoblot analysis. The membrane from rat inner medulla was prepared as described previously (19). About $5 \mu \mathrm{g}$ of the protein and in vitro synthesized ClC-K1 protein were separated on SDS-PAGE. The proteins were then blotted onto nitrocellulose (Hybond-ECL; Amersham) by semi-dry electroblotting for $1 \mathrm{~h}$. The blots were air dried and then blocked overnight at $4^{\circ} \mathrm{C}$ with $2 \%$ nonfat dry milk in phosphatebuffered saline (PBS) $\left(80 \mathrm{mM} \mathrm{Na} \mathrm{HPO}_{4}, 20 \mathrm{mM} \mathrm{NaH} \mathrm{PO}_{4}, 100 \mathrm{mM}\right.$ $\mathrm{NaCl}, \mathrm{pH} 7.5$ ) with $0.1 \%$ Tween 20 (PBS-T). The blot were washed twice in PBS-T and incubated with the primary antibody for $1 \mathrm{~h}$ at $37^{\circ} \mathrm{C}$. The antiserum was diluted 1:200 in PBS-T or preabsorbed with the peptide antigen $(0.5 \mu \mathrm{g} / \mathrm{ml})$ in PBS-T at $4^{\circ} \mathrm{C}$ overnight. The blots were washed in PBS-T four times and the bound antibody was detected by ${ }^{125} \mathrm{I}$-protein $\mathrm{A}$.

Immunohistochemistry. Rat kidney was dissected and cut into pieces no greater that $0.5 \times 1.0 \times 1.0 \mathrm{~cm}$ and immediately fixed by immersion in a solution of $2 \%$ paraformaldehyde. Tissues were frozen, and sectioned onto poly-L-lysine-coated glass slides. After blocking nonspecific binding by incubating in 10\% goat serum in PBS for $15 \mathrm{~min}$, sections were incubated with 1:200 dilution of antisera at $37^{\circ} \mathrm{C}$ for $1 \mathrm{~h}$, then washed with PBS for 15 min three times. FITC-labeled anti-rabbit immunoglobulin antibody (1:100 dilution) was applied to the sections and incubated for $1 \mathrm{~h}$ at $37^{\circ} \mathrm{C}$. After three times wash with PBS, the sections were observed under a fluorescence microscopy. As a control, the first antibody was replaced with preimmune rabbit serum or antisera preabsorbed with synthetic antigen peptide.

For immunoelectron microscopy, frozen sections were incubated in methanol containing $0.3 \% \mathrm{H}_{2} \mathrm{O}_{2}$ to block endogenous peroxidase. After wash with PBS, the sections were incubated with non-immune $10 \%$ goat serum, then the primary antibody (1:100 dilution) was applied and incubated overnight at $4^{\circ} \mathrm{C}$. After wash with PBS, the peroxidase-labeled goat anti-rabbit IgG (FAb fraction; Biosys S.A., Compiegne, France) was added and incubated for $6 \mathrm{~h}$ at room temperature. The sections were extensively washed with PBS and the peroxidase reaction was

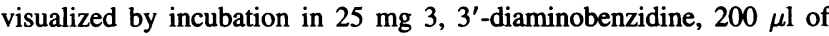

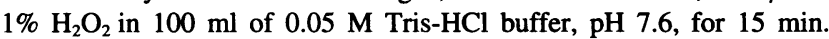
Stained sections were postfixed in $1 \%$ OsO4 for $30 \mathrm{~min}$, dehydrated in an ethanol series, and embedded in Quetol 812. Ultrathin sections were prepared and examined by Hitachi H-800 electron microscopy.

Functional expression in Xenopus oocytes. Preparation of Xenopus oocytes and CIC-K1 transcript were performed as described previously (11). About $10 \mathrm{ng}$ of ClC-K1 cRNA was injected into oocytes and incubated for 2-4 d in modified Barth solution (20). Two electrode voltage clamp was performed using pCLAMP software (Axon Instruments, Inc., Foster City, CA).

\section{Results}

Characterization of antisera. Two synthetic peptides ( $\mathrm{N}$ and $\mathrm{C}$ ) corresponding amino- and carboxy-terminal portions of $\mathrm{ClC}$ $\mathrm{K} 1$ protein were used as antigens. Five rabbits were immunized for each peptide. ELISA was used to screen the titer of antisera. The titer of an antiserum $(\mathrm{N}-1)$ for peptide $\mathrm{N}$ was $12,000: 1$ and those of two antisera $(\mathrm{C}-3,4)$ for peptide $\mathrm{C}$ were $24,000: 1$. As shown in Fig. $1 a$, the apparent molecular mass of in vitro synthesized $\mathrm{ClC}-\mathrm{K} 1$ protein was $60 \mathrm{kD}$. Calculated molecular mass from amino acid sequence was $76 \mathrm{kD}$. This discrepancy sometimes happens in case of membrane protein (21). C-3 could recognize the in vitro synthesized protein at 1:200 dilution (Fig. $1 \mathrm{~b}$ ). Pretreatment of the antibody with the antigen peptide completely abolished the band, indicating that C-3 specifically recognized $\mathrm{ClC}-\mathrm{K} 1$ protein (Fig. 1 b) . C-4 also recognized this band but the signal to noise ratio in the immunoblot analysis of kidney medulla was low and $\mathrm{N}-1$ did not recognize this protein (data not shown). Therefore, we used C-3 for immunoblot analysis of kidney medulla protein and immunohistochemistry. In vitro translation reaction in the presence of canine pancreatic microsome membrane yielded another band at $70 \mathrm{kD}$ in SDS-PAGE (Fig. $1 b$ ), suggesting that $\mathrm{ClC}-\mathrm{K} 1$ protein could be glycosylated.

Immunoblot analysis of $\mathrm{ClC}-\mathrm{K} 1$ in kidney. $\mathrm{C}-3$ recognized the protein in kidney papilla whose apparent molecular mass was the same $(70 \mathrm{kD})$ as that of $\mathrm{ClC}-\mathrm{K} 1$ protein synthesized in vitro in the presence of canine pancreatic microsome membrane. The preabsorption of the antibody made the band disappear (Fig. 2), demonstrating the specificity of $70-\mathrm{kD} \mathrm{ClC}-\mathrm{K} 1$ protein in the inner medulla of rat kidney.

Immunohistochemistry. C-3 antiserum recognized the thin limb of Henle's loop in inner medulla of rat kidney (Fig. $3 \mathrm{c}$ ). Preabsorbed antibody did not recognize the thin limb, suggesting the specificity of the staining (Fig. $3 d$ ). Collecting dusts were not stained. There was no specific staining in cortex and outer medulla, either (Fig. 3, $a$ and $b$ ). Higher magnification of inner medulla demonstrated that the staining in the thin limb was observed in the region of plasma membrane (Fig. $4 a$ ). Furthermore, the nuclei (indicated by arrow in Fig. $4 a$ ) were 

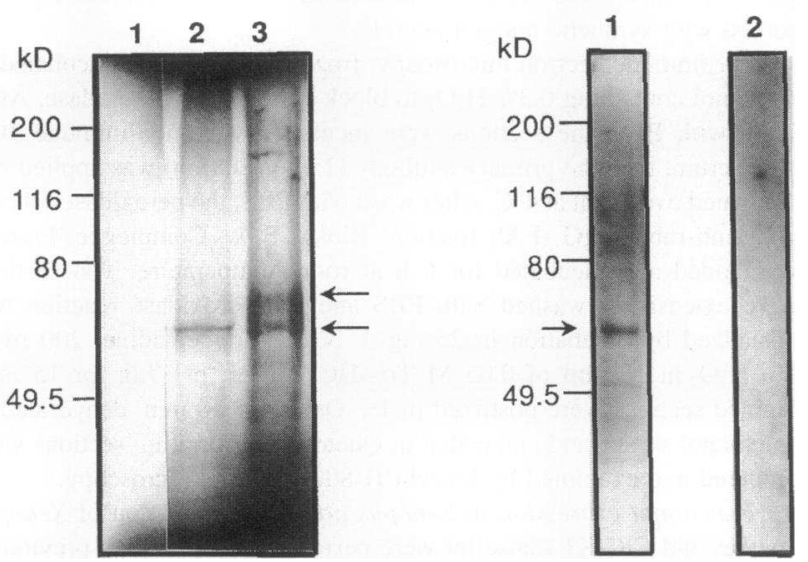

Figure 1. (a) In vitro translation of $\mathrm{ClC}-\mathrm{K} 1$ protein in the presence or absence of canine pancreatic microsome membrane. (Lane 1) No RNA was added to the in vitro translation reaction. (Lanes 2 and 3) In vitro-synthesized capped ClC-K1 cRNA was used as a template for in vitro translation in the absence (lane 2) or presence (lane 3 ) of canine pancreatic microsome membrane. Arrows indicate the in vitro translation products. (b) Specificity of the polyclonal antibody (C-3) to ClC$\mathrm{K} 1$ protein. (Lanes 1 and 2) ClC-K1 protein synthesized in vitro with cold methionine was separated in SDS-PAGE (4-20\% gradient gel) and blotted on nitrocellulose filter. The filter was cut into pieces; C-3 antibody (1:200 dilution) (lane 1 ) or the preabsorbed C-3 antibody (lane 2) was used for immunoblot analysis as a primary antibody. ${ }^{125}$ I-protein A was used for the detection of bound antibody. The strips of blots were visualized by autoradiography.

surrounded by the staining, suggesting that both apical and basolateral plasma membranes were stained. To confirm the localization of ClC-K1 protein, immunoelectron microscopy was performed. As indicated in Fig. $4 b, \mathrm{ClC}-\mathrm{K} 1$ protein was located in the region of both apical and basolateral plasma membranes.

There are two components of thin limbs in inner medulla, descending (tDL) and ascending limb of Henle's loop (tAL). Previous perfusion studies revealed that tAL has an extremely high transepithelial chloride permeability, but nominally zero water permeability (14). In contrast, tDL has high osmotic water permeability but much less chloride permeability compared to tAL (22). Recently, aquaporin-CHIP (AQP-CHIP) water channel was found to be localized in proximal tubules and tDL and may be responsible for high water permeability in those nephron segments. Because AQP-CHIP is not present in tAL, we could determine whether the thin limb in inner medulla stained by ClC-K1 antibody was ascending or descending limb. Fig. 5, $a$ and $b$ show the staining of the section of inner medulla by $\mathrm{ClC}$ K1 and AQP-CHIP antibody (a generous gift from Dr. P. Agre, John Hopkins University, Baltimore, MD), respectively. As shown in Fig. $5 b$, the number of descending limb in the section near the tip of papilla was smaller that of ascending limb (Fig. $5 \mathrm{a}$ ). This is probably due to the fact that the ascending limbs begin before the bend at the tip of papilla. This was consistent with the findings of Chou et al. (23). By comparing the staining pattern (Fig. 6, $a-c$ ) with two antibodies, the thin limbs stained by ClC-K1 were apparently different from the thin limbs recognized by AQP-CHIP, demonstrating that $\mathrm{ClC}-\mathrm{K} 1$ is localized in the thin ascending limb of Henle's loop. The staining of the

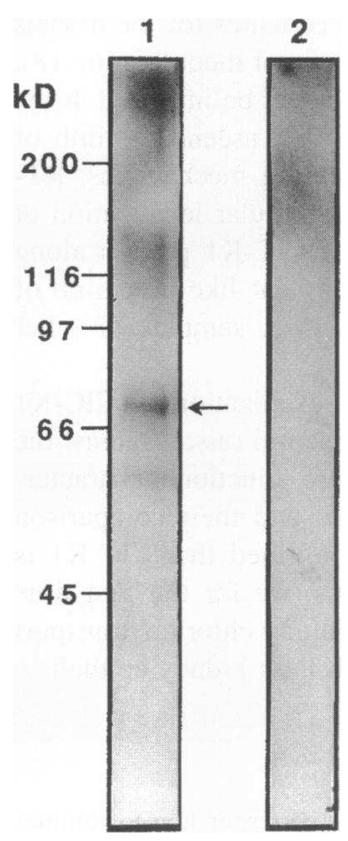

Figure 2. Immunoblot analysis of the membrane protein in rat kidney medulla. $5 \mu \mathrm{g}$ of membrane proteins from rat kidney medulla was separated on SDS-PAGE and blotted on nitrocellulose membrane. 1:200 dilution of $\mathrm{C}-3$ antibody (lane 1) and the preabsorbed C-3 antibody (lane 2) were used as primary antibodies.

sections (Fig. 5, $c$ and $d$ ) of outer medulla where only tDL exists also demonstrated that $\mathrm{ClC}-\mathrm{K} 1$ is not present in the descending limb.

Functional characterization of $\mathrm{ClC}-\mathrm{K} 1$ in Xenopus oocytes. We have previously demonstrated that $\mathrm{ClC}-\mathrm{K} 1$ is a chloride channel (11), however further functional characterization should be necessary to elucidate its physiological significance. First, we tested the effect of extracellular $\mathrm{pH}$ on the expressed chloride current because decrease of bath $\mathrm{pH}$ significantly reduced the transepithelial chloride flux in tAL $(15,24)$. As shown in Fig. $7 a$, the lowering of extracellular pH dramatically reduced the expressed chloride current. Hill plot analysis (Fig. 7 b) showed that the coefficient was 1.2 , suggesting the interaction of $\mathrm{H}^{+}$ and $\mathrm{Cl}^{-}$was 1 to 1 . Next the effect of extracellular calcium on the expressed current was determined. Removal of extracellular calcium chloride from ND 96 significantly reduced the overall current (Fig. 7 c), which is consistent with the in vitro perfusion study in tAL (25). This effect was reversed by the re-addition of calcium to ND96. The injection of $40 \mathrm{nl}$ of $100 \mathrm{mM}$ EGTA into oocytes did not affect the expressed current, suggesting that the effect of extracellular calcium was not mediated by intracellular calcium concentration. Finally, the effects of drugs known to modulate transcellular chloride transport in tAL were tested in oocytes. Fig. $8 a$ shows the dose response of furosemide on the expressed chloride currents in oocytes. Furosemide inhibited the expressed chloride current dose dependently (Fig. $8 a$ ). $K_{i}$ was $100 \mu \mathrm{M}$. This inhibitory effect could be washed out immediately. Another agent we tested in this study was $N$ ethyl-maleimide (NEM). This agent was reported to have dual effects on the transepithelial chloride transport in tAL (26). NEM (1 mM) inhibited chloride flux in tAL irreversibly, but re-addition of NEM to the pretreated tubules stimulated the chloride transport (26). We could not apply $1 \mathrm{mM} \mathrm{NEM}$ because it affected the basal oocyte permeability significantly. NEM (0.1 $\mathrm{mM}$ ) did not affect the ion permeability in $\mathrm{H}_{2} \mathrm{O}$-injected oocytes but stimulated the expressed chloride current about $100 \%$ (Fig. $8 b$ ). This effect was reversible. 

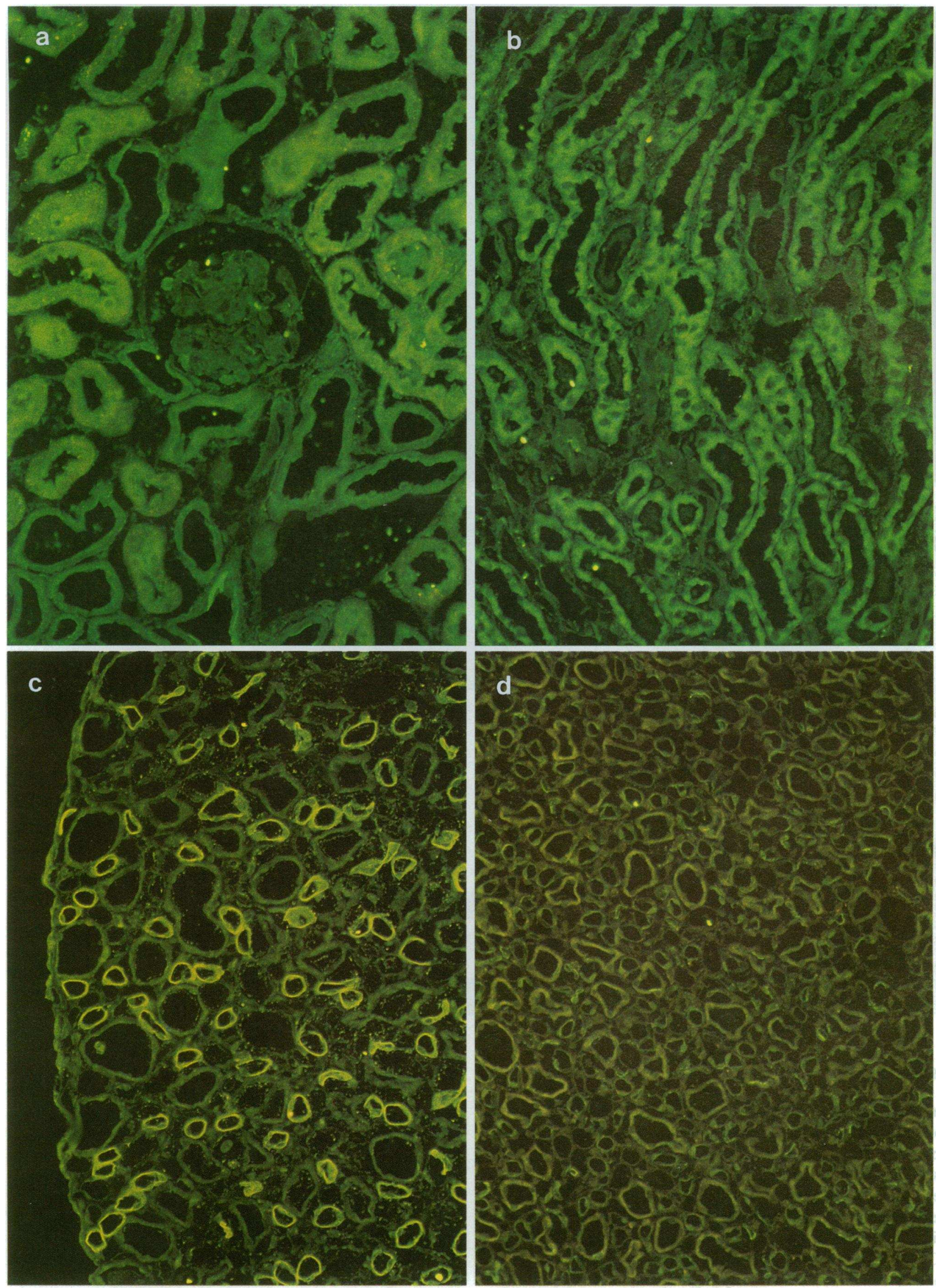

Figure 3. Immunohistochemical localization of ClC-K1 in rat kidney. (a) From cortex; $(b)$ from outer medulla; and $(c)$ from inner medulla. In $a$, $b$, and $c, 1: 200$ dilution of C-3 antiserum was used. $(d)$ Preabsorbed C-3 was used instead of C-3. $(\times 140)$. 

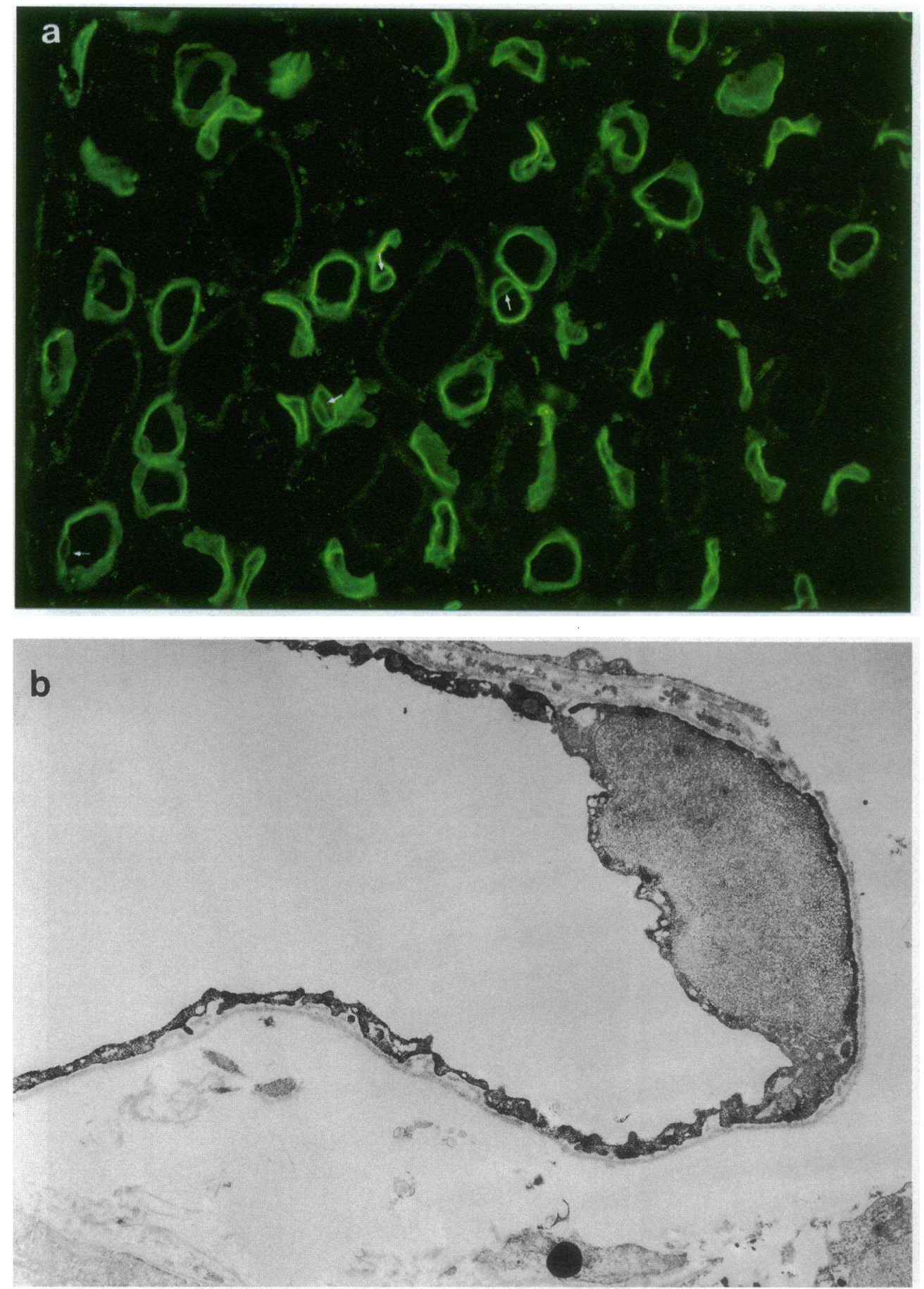

Figure 4. Higher magnification of rat inner medulla $(a)$ and immunoelectron microscopy of the thin limb of Henle's loop (b). (a) Higher magnification $(\times 320)$ of rat inner medulla. Staining was only observed in the thin limb of Henle's loop in inner medulla. Arrows indicate the staining of apical plasma membrane in the thin limb. (b) Arrows indicate the staining with $\mathrm{ClC}-\mathrm{K} 1$ antibody. $(\times 4,000)$.

\section{Discussion}

Here we could clearly demonstrate the localization of ClC-K1 protein. Specificity of the antiserum was confirmed by immunoblot analysis using in vitro translated $\mathrm{ClC}-\mathrm{K} 1$ protein as a positive control. The calculated molecular mass is $76 \mathrm{kD}$, however the apparent molecular mass in SDS-PAGE was $60 \mathrm{kD}$. The difference of apparent molecular weight from the calculated weight could be due to the posttranslational modification of $\mathrm{ClC}-\mathrm{K} 1$ protein in vivo. In a case of the electroplax sodium channel, which binds unusually high amounts of SDS due to posttranslational acquisition of hydrophobic residues (i.e., cova- lently bound fatty acids or phopholipids), has a high electrophoretic mobility and thus smaller apparent size compared with standard proteins in SDS-PAGE (27). The same phenomenon was also reported in $\mathrm{Na} / \mathrm{H}$ exchanger (21). In vitro synthesis of $\mathrm{ClC}-\mathrm{K} 1$ protein in the presence of canine pancreatic microsome membrane yielded another band at $70 \mathrm{kD}$ (Fig. 1 b). In immunoblot analysis of rat inner medulla, $\mathrm{ClC}-\mathrm{K} 1$ protein appeared to exist at $70 \mathrm{kD}$. These results suggest that the majority of $\mathrm{ClC}-\mathrm{K} 1$ protein is glycosylated in vivo.

RT-PCR using dissected nephron segments demonstrated that the expression of $\mathrm{ClC}-\mathrm{K} 1$ was also noted in $\mathrm{TDL}$ and collecting ducts as well as tAL (11). This was probably due to the 

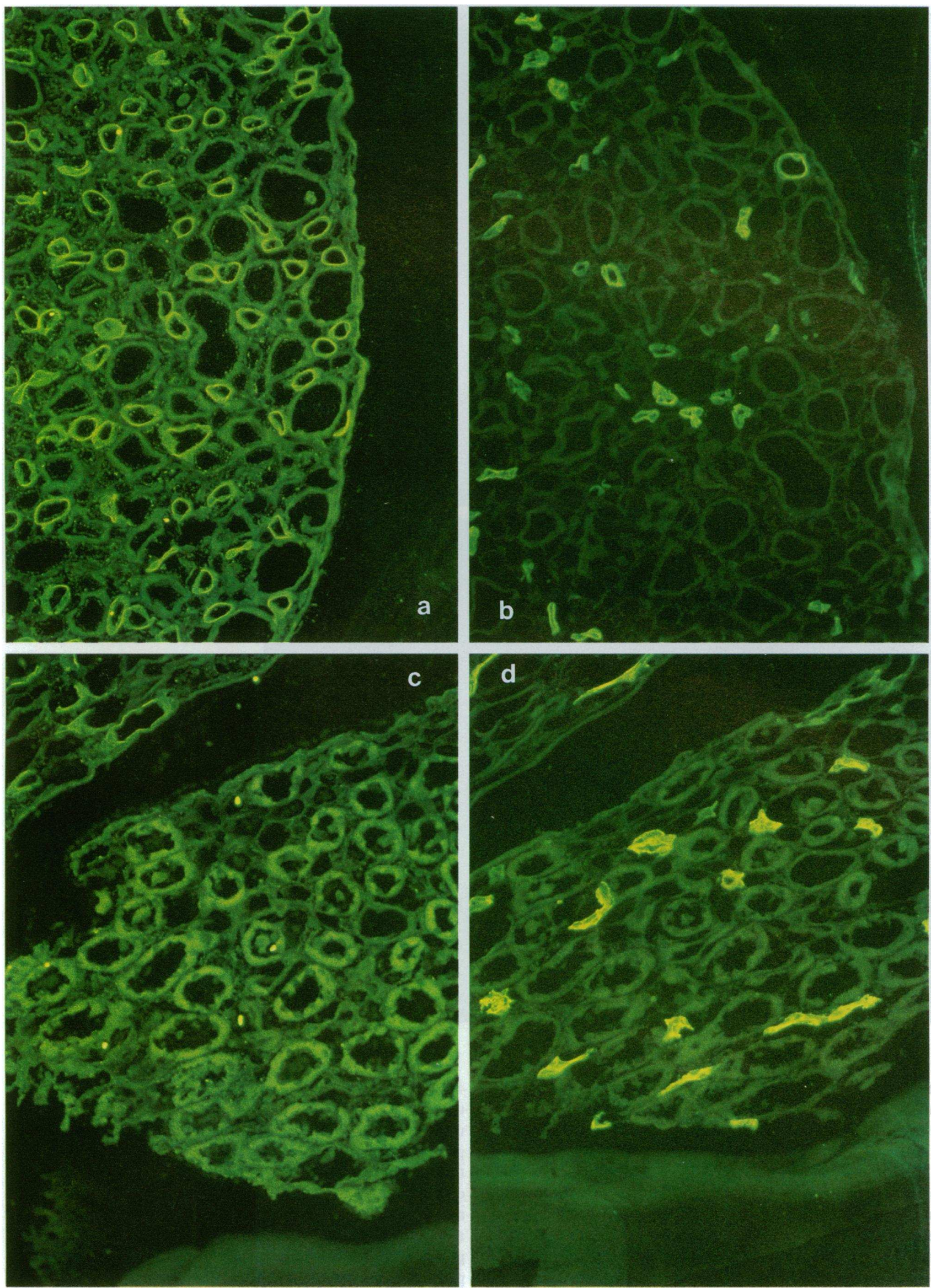

Figure 5. Comparison of the localization between ClC-K1 ( $a$ and $c$ ) and AQP-CHIP ( $b$ and $d$ ) in inner medulla and outer medulla. Sequential sections were incubated with anti-ClC-K1 (1:200) and anti-CHIP28 (1:500) and visualized by FITC-conjugated anti-rabbit IgG goat antibody (1:100). $a$ and $b$ from inner medulla and $c$ and $d$ from outer medulla. $(\times 140)$. 

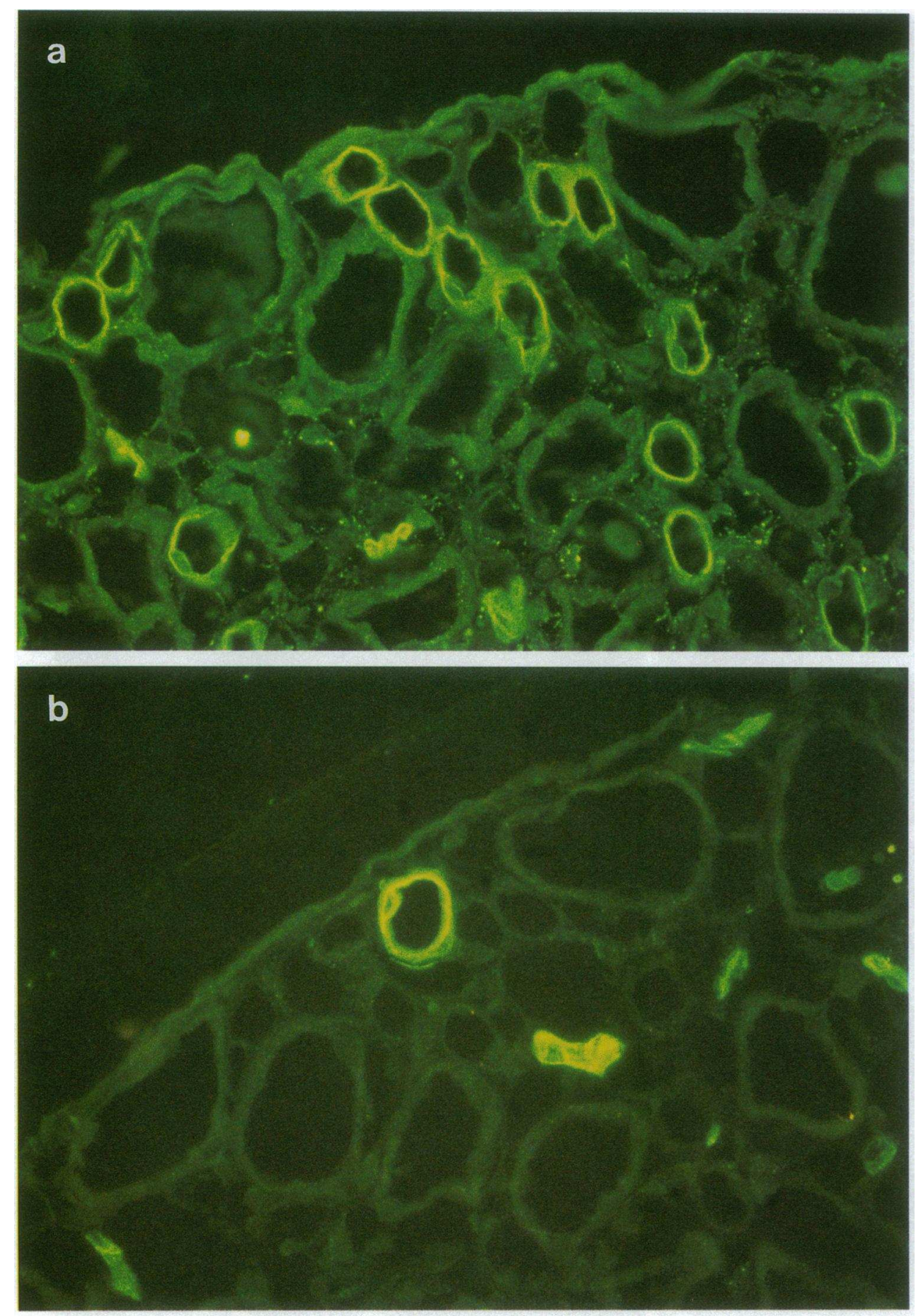

Figure 6. Higher magnification of the staining in the inner medulla with anti-ClC-K1 $(a)$ and anti-CHIP28 (b). Fig. $6 c$ illustrates the staining patterns observed in $a$ and $b .(\times 320)$.

contamination of tiny fragments of tAL into the other PCR samples. The exclusive localization of $\mathrm{ClC}-\mathrm{K} 1$ in $\mathrm{tAL}$ suggested that other chloride channels might be present in the other nephron segments such as the thick ascending limb of Henle's loop and the cortical collecting duct, where the basolateral localization of chloride channels has been demonstrated by microperfusion and patch clamp studies (4). *Immunofluorescence study revealed the existence of $\mathrm{ClC}-\mathrm{K} 1$ on the both sides of the plasma membrane. Because both membranes are close to each other, we could recognized the existence of the protein on the apical surface only at the sites of nuclei by immunofluorescence (Fig.
4 a). However, immunoelectron microscopy showed the existence of $\mathrm{ClC}-\mathrm{K} 1$ protein in the other part of apical plasma membrane (Fig. $4 b$ ). ClC-K1 expressed in oocytes showed no gating mechanisms and open at any membrane voltage. Although there was a slight outward rectification, chloride ion could pass through this channel in both way. The existence of a channel of such properties on both plasma membranes of a nephron segment makes the transcellular chloride transport possible in tAL, which is consistent with the highest chloride permeability measured by the perfusion study (14).

The localization of $\mathrm{ClC}-\mathrm{K} 1$ protein and the previous in vitro 


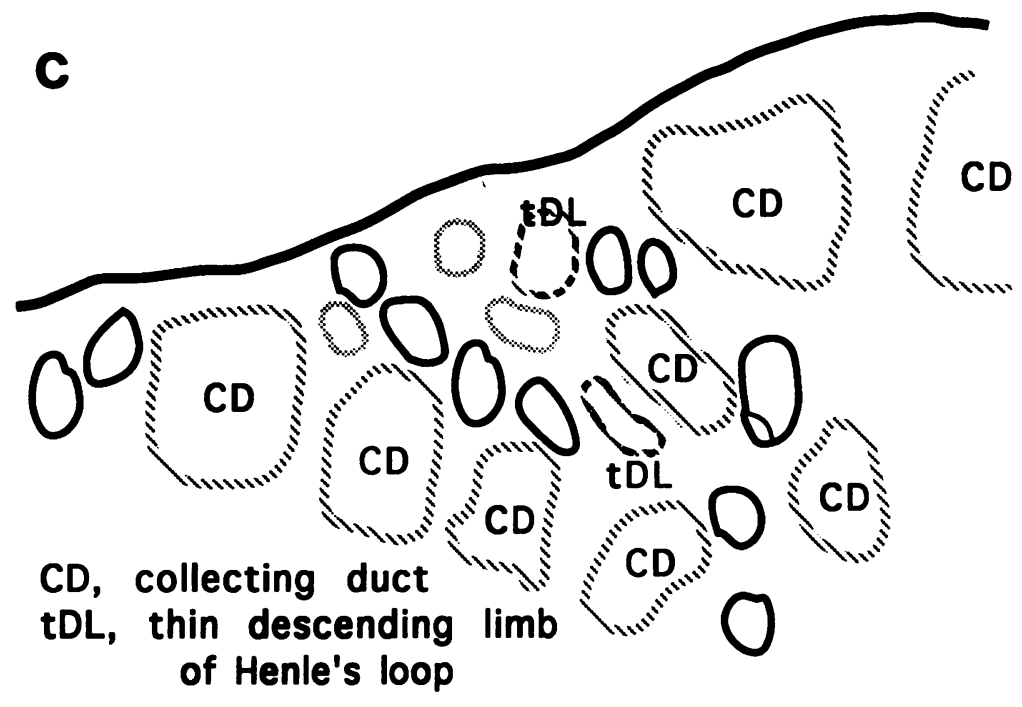

Figure 6 (Continued)

perfusion studies strongly suggested that $\mathrm{ClC}-\mathrm{K} 1$ is a major chloride channel in tAL. To confirm this, we next determined the functional characterization of ClC-K1 to see whether or not the transport characteristics of the expressed chloride current in oocytes would be consistent with those previously characterized in the perfused tAL. We have previously characterized the inhibitor sensitivity and anion selectivity. DIDS (4,4'-diisothiocyanatostilbene-2,2'-disulphonic acid) was effective $\left(K_{i}=30\right.$ $\mu \mathrm{M})$, but 9 -AC (9-anthracene carboxylic acid) was less effective. Anion selectivity sequence was $\mathrm{Br}>\mathrm{Cl} \gg \mathrm{I} \gg$ cyclamate $=$ gluconate. These data are consistent with the data from the in vitro perfusion studies $(28,29)$. In this study, we further characterized the expressed current. In the microperfusion experiments of tAL, low extracellular $\mathrm{pH}$ decreased the transcellular chloride flux almost completely $(15,24)$. The expressed current in oocytes was also regulated by extracellular $\mathrm{pH}$ (Fig. $7 \mathrm{a}$ ). Hill coefficient in our study was 1.2 (Fig. $7 \mathrm{~b}$ ), indicating that the interaction of $\mathrm{H}^{+}$and $\mathrm{Cl}^{-}$could be $1: 1 . K_{d}$ is 6.9 in this study (Fig. $7 b$ ) and 6.3 in the perfusion study (24). Kondo et al. reported the pH-dependent regulation was only observed at $37^{\circ} \mathrm{C}$, not at $22^{\circ} \mathrm{C}$, and Hill coefficient was 2.0. We could observe the effect of low $\mathrm{pH}$ at room temperature. These discrepancies cannot be explained completely at present, but probably are due to the difference in methods of measuring chloride transport or the species difference between hamster and rat. They recorded the transepithelial diffusion voltage as a measure for chloride flux when $\mathrm{Cl}^{-}$gradient was imposed (24-26, 29, 30 ), which is not a direct measurement of chloride transport. It is possible that what they measured might be influenced by other factors, such as cell metabolism. The physiological significance of $\mathrm{pH}$ regulation of this channel remains unclear now, because the small changes of $\mathrm{pH}$ under the physiological circumstances would not affect the current very much.

The other regulation observed in the perfusion studies was that by calcium ion. The reduction of extracellular calcium to nominally zero decreased the transepithelial diffusion voltage in the perfused tAL to $60 \%$ (25). The same level of inhibition was also observed in our study (Fig. $7 c$ ). Intracellular chelating of calcium by EGTA injection did not affect the current (Fig. $7 c$ ), indicating that the regulation by calcium was mediated by extracellular domain of ClC-K1.
Sensitivities to drugs other than DIDS and DPC were also verified. Furosemide, a potent inhibitor of $\mathrm{Na}, \mathrm{K}, 2 \mathrm{Cl}$-cotransporter, shown to inhibit chloride flux in tAL at $1 \mathrm{mM}$, also inhibited the expressed current $70 \%$ (30). We carefully measured the dose-response of furosemide on the expressed current and found that $K_{i}$ was $100 \mu \mathrm{M}$ (Fig. $8 a$ ). The concentration of furosemide in inner medulla could be as high as this level when a large dose of intravenous injection of furosemide is performed. Chloride diuresis by furosemide is thought to be attained only by the inhibition of chloride entry through $\mathrm{Na}, \mathrm{K}, 2 \mathrm{Cl}$-cotransporter on the apical membrane of the thick ascending limb (TAL), but we speculate the effect of furosemide on ClC-K1 also contribute to that because the water permeability in tAL is very low like that in TAL. In case of NEM, we could only observe the stimulatory effect on the chloride current (Fig. 8 b). Because Imai et al. (26) did not show the effect of $0.1 \mathrm{mM}$ NEM, we could not compare our result directly with theirs. Based on the experiment using NEM analog that does not permeate into cells, they speculated that the inhibitory effect might be mediated on intracellular site, and that the stimulatory effect might be mediated on the extracellular site (26). It is possible that NEM at $0.1 \mathrm{mM}$ could not get into oocytes.

Although there were some functional differences in details between the perfusion studies and the oocyte expression study, we think that these two chloride transport systems have basically the same characteristics. Accordingly, we conclude that $\mathrm{ClC}-\mathrm{K} 1$ is a major chloride channel in tAL and is responsible for transcellular transport in tAL. Chloride transport in tAL has been thought to be an important component of counter current system in inner medulla for urinary concentration (16-18). Although the exact mechanisms of passive model in counter current system are not yet clarified in any proposed models (1618), rapid chloride flux from luminal fluid in tAL to the surrounding interstitial space is necessary for making hypertonic environment in inner medulla. Under this circumstance, the opening of collecting duct water channel would facilitate the water reabsorption, thus lead to urinary concentration. These informations suggest the physiological significance of ClC-K1 chloride channel in urinary concentrating mechanisms. The discovery of highly specific inhibitors to ClC-K1 and the generation of ClC-K1 deficient animal would demonstrate the physiological role of $\mathrm{ClC}-\mathrm{K} 1$ more clearly. 
a

$\mathrm{pH} 7.57$

pH6.59

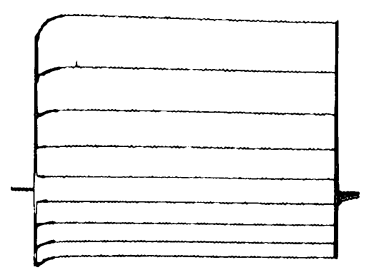

pH7.24

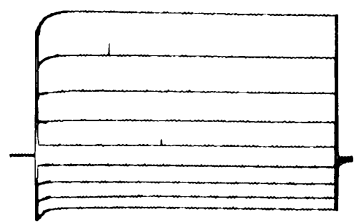

$\mathrm{pH} 7.14$
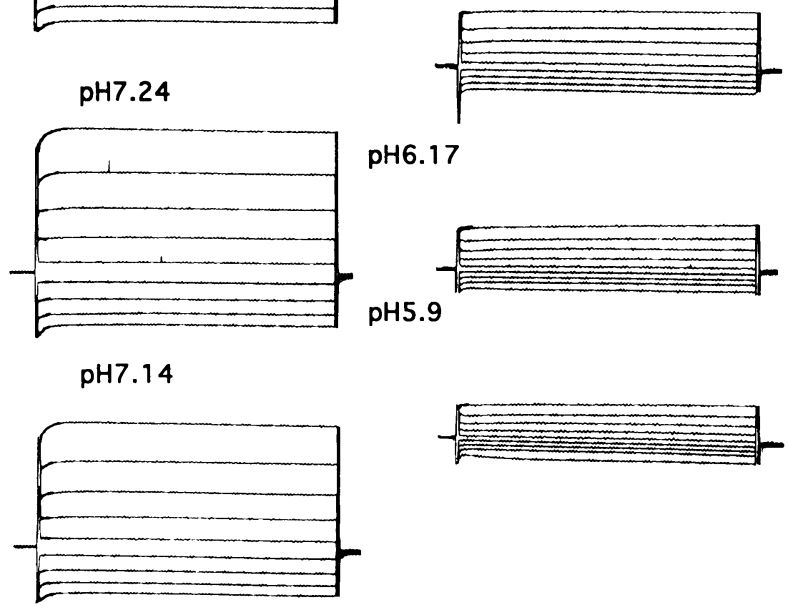

pH6.17

pH5.9

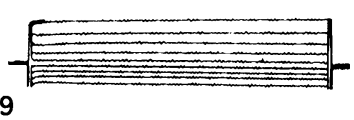

b
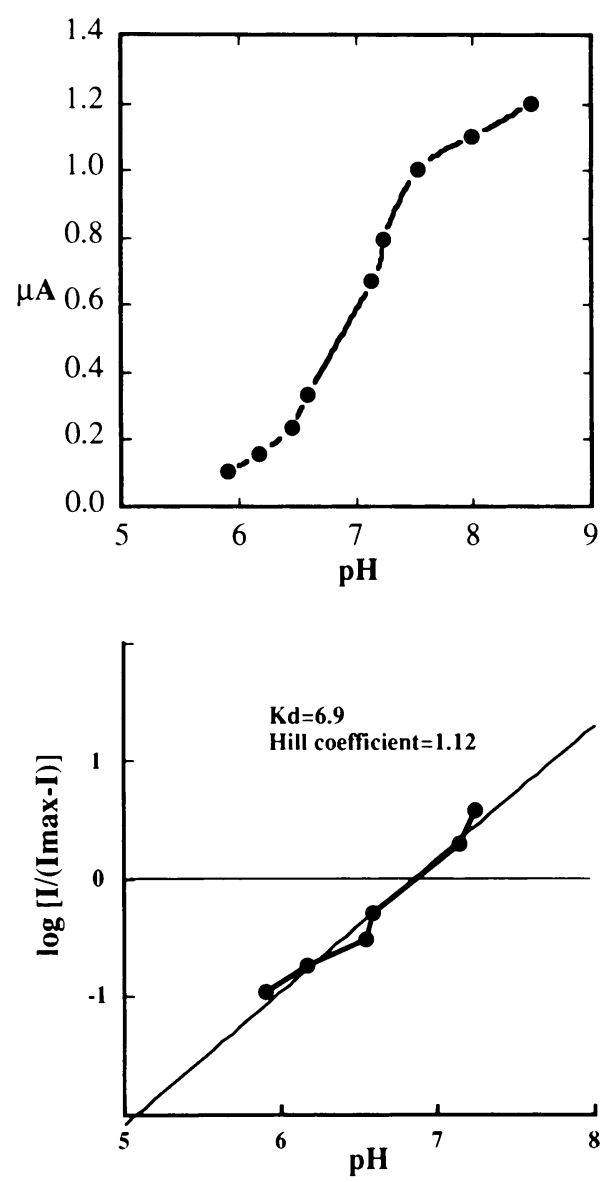

C

ND96

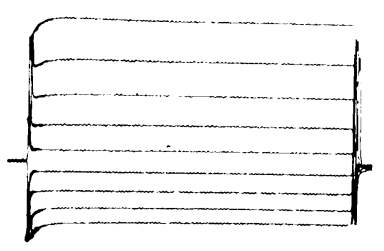

EGTA injected

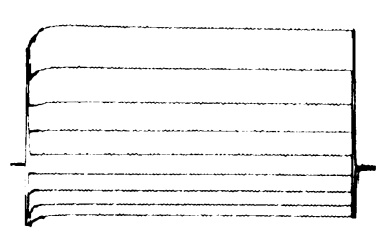

$\mathrm{Ca}(-) \mathrm{ND} 96$

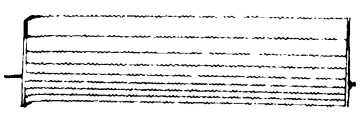

$100 \mathrm{nA}$

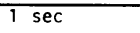

Figure 7. Effect of extracellular $\mathrm{pH}(a$ and $b$ ) and calcium $(c)$ on the expressed chloride current by ClC-K1 cRNA. a. Traces of chloride current elicited by $\mathrm{ClC}-\mathrm{K} 1 \mathrm{cRNA}$ in oocytes at different extracellular $\mathrm{pH}$. From the holding potential at $-30 \mathrm{mV}$, voltage was shifted to -100 to $+20 \mathrm{mV}$ at $20 \mathrm{mV}$ interval and clamped for $2 \mathrm{~s}$ at each voltage. b. upper panel, currents measured at $+40 \mathrm{mV}$ were plotted as a function of $\mathrm{pH}$, lower panel, Hill plot of the expressed chloride current as a function of $\mathrm{pH}$. (c) Extracellular calcium was reduced to nominally zero (ND96 Ca-), or 40 $\mathrm{nl}$ of $100 \mathrm{mM}$ EGTA was injected (EGTA injected). Lower panel shows the comparison of the current measured at $+40 \mathrm{mV}$. 
a

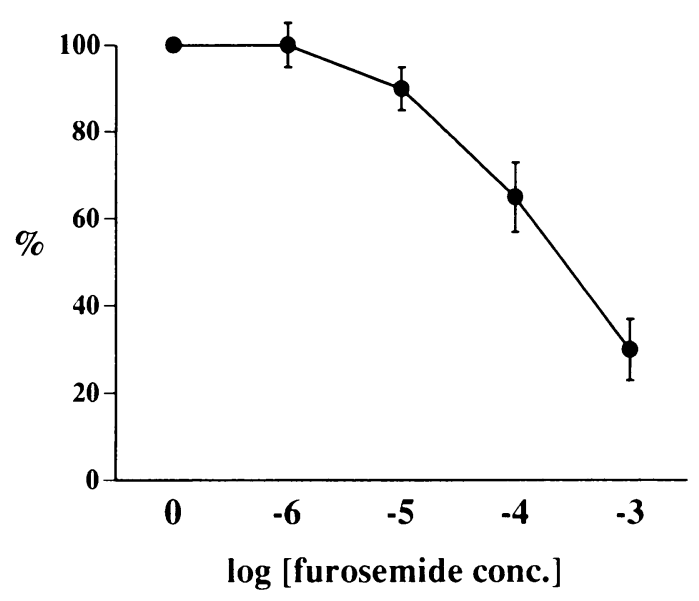

b

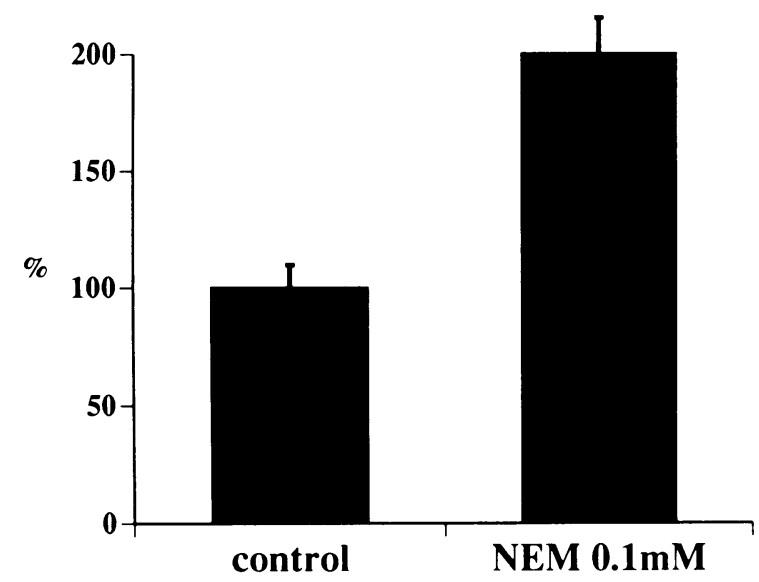

Figure 8. Effect of furosemide ( $a$ ) and NEM $(b)$ on the expressed chloride current in oocytes. (a) Dose-response curve of furosemide on the expressed chloride current in oocytes. The current was measured at $+40 \mathrm{mV} 1 \mathrm{~min}$ after the addition of furosemide. The effect of furosemide was reversible. (b) $0.1 \mathrm{mM}$ NEM did not affect the leak current in oocytes. The effect of NEM was reversible.

In summary, we could demonstrate the existence of the transcellular chloride transport pathway in $\mathrm{TAL}$ by clarifying the localization and the functional characterization of $\mathrm{ClC}-\mathrm{K} 1$ chloride channel protein.

\section{Acknowledgments}

We thank Professor Joseph S. Handler for critical reading of the manuscript.

This study was supported by a grant-in-aid from the Ministry of Education, Science, and Culture, Japan, the grant from the Ichiro Kanehara Foundation, and the grant from the Mitsubishi Foundation.

\section{References}

1. Halm. D. R., G. R. Rechkemmer. R. A. Schoumacher, and R. A. Frizzell, 1988. Apical membrane chloride channels in a colonic cell line activated by secretory agonists. Am. J. Physiol. 254:C505-C511.

2. McCann, J. D.. and M. J. Welsh. 1990. Regulation of Cl- and K+ channels in airway epithelium. Annu. Rev: Physiol. 52:115-135.

3. Greger, R. 1985. Ion transport mechanisms in the thick ascending limb of Henle’s loop of mammalian nephron. Physiol. Rev. 65:760-797.
4. Reeves, W. B., and T. E. Andreoli. 1992. Renal epithelial chloride channels Annu. Rev. Physiol. 54:29-50.

5. Jentsch, T. J., K. Steinmeyer, and G. Schwarz. 1990. Primary structure of Torpedo marmorata chloride channel isolated by expression cloning in Xenopus oocytes. Nature (Lond.). 348:510-514.

6. Landry. D.. S. Sullivan, M. Nicolaides, C. Redhead, A. Edelman, M. Field Q. Al-Awqati. and J. Edwards. 1993. Molecular cloning and characterization of p64, a chloride channel protein from kidney microsomes. J. Biol. Chem. 268:14948-14955.

7. Paulmichl, M., Y. Li. K. Wickman, M. Ackerman, E. Peralta, and D. Clapham. 1992. New mammalian chloride channel identified by expression cloning. Nature (Lond.). 356:238-241.

8. Riorden, J. R.. J. M. Rommens. B. -S. Kerem. N. Alon, R. Rozmahel, Z. Grzelczak. J. Zielenski, S. Lok. N. Plavsic, J. -L. Chou. M. L. Drumm, M. C. Lannuzzi. F. S. Collins, and L. -C. Tsui. 1989. Identification of the cystic fibrosis gene: cloning and characterization of complementary DNA. Science (Wash. DC). 245:1066-1073.

9. Steinmeyer, K., C. Ortland, and T. J. Jentsch. 1991. Primary structure and functional expression of a developmentally regulated skeltal muscle chloride channel. Nature (Lond.). 354:301-303.

10. Thiemann. A., S. Grunder, M. Pusch. and T. J. Jentsch. 1992. A chloride channel widely expressed in epithelial and non-epithelial cells. Nature (Lond.). 356:57-60

11. Uchida, S., S. Sasaki, T. Furukawa, M. Hiraoka, T. Imai, Y. Hirata, and F. Marumo. 1993. Molecular cloning of a chloride channel that is regulated by dehydration and expressed predominantly in kidney medulla. J. Biol. Chem. 268:3821-3824

12. Ishibashi, K.. S. Sasaki. S. Uchida, T. Imai, and F. Marumo. 1993. Tissue expression of mRNA of a chloride channel from MDCK cells and its regulation by protein kinases. Biochem. Biophys. Res. Commun. 192:561-567.

13. Krapivinsky, G. B.. M. J. Ackerman, E. A. Gordon, L. D. Krapivinsky, and D. E. Clapham. 1994. Molecular characterization of a swelling-induced chloride conductance regulatory protein, plCln. Cell. 76:439-448.

14. Imai, M and J. P Kokko 1974. Sodium, chloride, urea, and wate transport in the thin ascending limb of Henle. Generation of osmotic gradients by passive diffusion of solutes. J. Clin. Invest. 53:393-402.

15. Yoshitomi, K.. Y. Kondo, and M. Imai. 1988. Evidence for conductive $\mathrm{Cl}^{-}$pathways across the cell membranes of the thin ascending limb of Henle's loop. J. Clin. Invest. 82:866-871.

16. Imai, M.. J. Taniguchi, and K. Tabei. 1987. Function of thin loops of Henle. Kidney Int. 31:565-579.

17. Kokko, J. P., and F. C. Rector, Jr. 1972. Countercurrent multiplication system without active transport in inner medulla. Kidney Int. 2:214-223.

18. Stephenson, T. J. 1972. Concentration of urine in a central core model of the renal counterflow system. Kidney Int. 2:85-94.

19. Sasaki. S., K. Fushimi. H. Saito. S. Uchida, K. Ishibashi, M. Kuwahara T. Ikeuchi, K. Inui, K. Nakajima. T. -X. Watanabe, and F. Marumo. 1994. Cloning. characterization, and chromosomal mapping of human aquaporin of collecting duct. J. Clin. Invest. 93:1250-1256.

20. Uchida, S., H. M. Kwon. A. S. Preston, and J. S. Handler. 1991. Expression of Madin-Darby canine kidney cell $\mathrm{Na}^{+}$- and $\mathrm{Cl}^{-}$-dependent taurine transporter in Xenopus laevis oocytes. J. Biol. Chem. 266:9605-9609.

21. Biemesderfer, D., J. Pizzonia. A. Abu-Alfa, M. Exner, R. Reilly, P. Igarashi, and P. S. Aronson. 1993. NHE3: a Na+/H+ exchanger isoform of renal brush border. Am. J. Physiol. 265:F736-F742.

22. Imai. M.. J. Taniguchi, and K. Yoshitomi. 1988. Transition of permeability properties along the descending limb of long-loop nephron. Am. J. Physiol. 254:F323-F328.

23. Chou. C. -L., S. Nielsen, and M. A. Knepper. 1993. Structural-functiona correlation in chinchilla long loop of Henle thin limb: a novel papillary subsegment. Am. J. Phvsiol. 265:F863-F874.

24. Kondo. Y., K. Yoshitomi, and M. Imai. 1987. Effect of $\mathrm{pH}$ on $\mathrm{Cl}^{-}$transpor in TAL of Henle's loop. Am. J. Physiol. 253:F1216-F1222.

25. Kondo, Y.. K. Yoshitomi, and M. Imai. 1988. Effect of $\mathrm{Ca}^{2+}$ on $\mathrm{C}$ transport in thin ascending limb of Henle's loop. Am. J. Physiol. 254:F232-F239.

26. Imai. M., Y. Kondo. C. Koseki, and K. Yoshitomi. 1988. Dual effect of

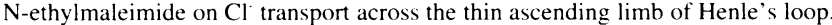
Pflugers Arch. 411:520-528.

27. Thornhill. W. B., and S. R. Levinson. 1987. Biosynthesis of electroplax sodium channel in Electrophorus electrocytes and Xenopus oocytes. Biochemistry: $26: 4381-4388$

28. Isozaki, T., K. Yoshitomi, and M. Imai. 1991. Selectivity of ion permeability across ascending thin limb of Henle's loop: Interaction of $\mathrm{Cl}$ - and other halogens with anion transport system. Kidney Int. 40:S113-S118.

29. Kondo, Y., K. Yoshitomi, and M. Imai. 1987. Effects of anion transport inhibitors and ion substitution on $\mathrm{Cl}^{-}$transport in TAL of Henle's loop. Am. J. Physiol. 253:F1206-F1215.

30. Kondo, Y., K. Yoshitomi, and M. Imai. 1987. Effects of Cl- transport inhibitors on $\mathrm{Cl}$ - permeability across hamster ascending thin limb. Am. J. Physiol 253:F92-F98 